
$\$$ Research Square
Preprints are preliminary reports that have not undergone peer review.
They should not be considered conclusive, used to inform clinical practice,
or referenced by the media as validated information.

\title{
Root-associated endophytic fungal community composition and structure of three medicinal licorices in Xinjiang
}

Hanli Dang

Shihezi University

Zhongke Wang

Shihezi University

Guifang Li

Shihezi University

Yudi Mu

University of Wisconsin-Madison

Xinhua Lv

Shihezi University

Li Zhuang ( $\nabla$ 3033573705@qq.com)

Shihezi University

Research

Keywords: bioactive compounds, endophytic fungal community, glycyrrhiza, Soil physicochemical

Posted Date: September 29th, 2020

DOl: https://doi.org/10.21203/rs.3.rs-82470/v1

License: () (7) This work is licensed under a Creative Commons Attribution 4.0 International License. Read Full License 


\section{Abstract}

The purpose of this study was to explore the diversity and composition of endophytic fungal community in the root of three medicinal licorices, and learn more about its biological characteristics by analyzing its interaction with soil and root factors. A total of 2,118,633 effective sequences and 1,063 effective operational taxonomic units (OTUs) with $97 \%$ identity were obtained by high-throughput sequencing among 27 samples. In this study, a total of 8 phyla and 140 genera were annotated, among them, the phylum Ascomycota and Basidiomycota, and the genera Fusarium, Paraphoma and Helminthosporium were significantly dominant in the 27 samples. Wilcoxon rank sum test showed that the Shannon index was significantly different distribution between Glycyrrhiza uralensis and Glycyrrhiza inflata, especially $0-20 \mathrm{~cm}$ at the root depth, the Chao1 index in Glycyrrhiza inflate was significantly affected by root depth, and there were significant differences in beta diversity between Glycyrrhiza uralensis and Glycyrrhiza inflata. Distance-based redundancy analysis (db-RDA) showed that soil physicochemical properties (available potassium and ammonium nitrogen), and the root factor (liquiritin and water content) were the main contributing factors to the variations in the overall structure of endophytic fungal community in this study. This study provides useful information for formulating strategies to improve the quantity and quality of medicinal licorices.

\section{Introduction}

Glycyrrhiza species are perennial herbs with widely grows in arid and semi-arid regions [1]. There are three different original plants of Glycyrrhiza stipulated in Chinese Pharmacopeia, namely dried root and rhizome of Glycyrrhiza uralensis, Glycyrrhiza inflate and Glycyrrhiza glabra its dried roots and rhizomes is one of the most commonly used herbs medicines in both Eastern and Western countries [2]. A wide variety of bioactive compounds can be extracted from root [3], mainly include triterpene saponins, polysaccharides and flavonoids [4]. Glycyrrhizic acid, the richest content of triterpene saponins [5], is the important pharmacological bioactive compounds with anti-inflammatory [6], antiviral and immune regulation [7, 8] and other biological effects. Liquiritin is a major component of flavonoids that mainly exerts anti-inflammatory [9], antioxidant and antibacterial [10,11]. Because of its medicinal and economic value, Glycyrrhiza plant has become the research direction of medicinal Glycyrrhiza to improve the content of licorice herbal medicine and understand its ecological characteristics.

The traditional view widely believes that the quality and quantity of the bioactive compounds extracted from medicinal plants are largely affected by the genetic background of the related plant, the ecological environment in which the plant lives, and soil nutrients [12, 13]. However, in recent years, some studies $[14,15]$ have shown that endophytic fungi have played a very important role in influencing the quality and quantity of bioactive compounds of medicinal plants through specific fungus-host interactions.

Endophytes, especially endophytic fungi, are one of the most important components in plant micro-ecosystems [16]. Endophytic fungi can form symbiotic relationships with host plants, on the one hand, which can present and grow in different healthy tissues of living plants, including stems [17], leaves [18] and roots [19]. Endophytic fungi, on the other hand, can extract carbohydrates and other nutrients from the host plant for their own growth [20]. In return, host plants may receive benefits from endophytic fungi associations. First, endophytic fungi can promote the growth of host plants by increasing hormones, including Gibberellin, Indoleacetic acid, Abscisic acid, Zeatin [21]. Second, endophytic fungi can enhance the resistance of host plants to environmental stress by producing biologically bioactive compounds [22,23], such as, endophytic fungi of wheat can promote plant growth and abiotic stress resistance [24]. Last but not least, endophytic fungi can promote the accumulation of secondary metabolites of the host plant [25], such as paclitaxel and deoxypodophyllotoxin, thereby affecting the quantity and quality of bioactive compounds of medicinal plants.

Endophytic fungi have great biodiversity and are widely distributed in various terrestrial and aquatic plants species [26], and numerous studies have shown that endophytic fungi can be isolated from various plants species, ranging from important cash crop species [27] such as soybean, to medicinal plant species $[28,29]$, such as Dendrobium Officinzle and Sceletium Tortuosum. However, it should be noted that, with the rapid development of high-throughput sequencing technology and bioinformatics, a large number of undiscovered fungi have been discovered [30]. Previous studies based on high-throughput sequencing technology have speculated that there are as many as 5.1 million fungal species, most of which are involved in plant-endophytic interactions [31]. At present, only a small part of endophytic fungi are isolated and identified, and most of the endophytic fungi in medicinal plant cannot be purely cultured on the existing medium [32]. Therefore, it is necessary to detect the endophytic fungi community in medicinal plants by adopting non-culture methods. Modern molecular technology, especially Illumina high-throughput sequencing technology, is an emerging technology in recent years, which can comprehensively and accurately detect the diversity of endophyte communities in medicinal plants [33,34]. The high-throughput sequencing technique of next-generation sequencing is a more robust and accurate microbial community characterization technique compared to $18 \mathrm{~S}$ rDNA-based non-culture methods and conventional culturing methods.

Numerous studies [35] have shown that the host genetic background (genotype or species) determine the composition of endophytic fungi. Meanwhile, soil fertility and ecological environment directly affect the content of bioactive compounds of medicinal plants, which will indirectly affect the composition and community structure of endophytic fungi [16]. However, for now, there is little information about the composition of endophytic fungi in the root of medicinal licorices at different genetic backgrounds (species), and soil environmental factors affecting the community structure of endophytic fungi in the root of medicinal licorice are still unclear. Therefore, in this study, we investigated the distribution and composition of endophytes fungal species of three medicinal licorices at three root depths through high-throughput sequencing and explored their relationship with host plants' bioactive compounds and soil physicochemical properties. The results will enhance researchers' understanding about the environmental and host factors that influence endophytic fungi and the friendly relationship between endophytic fungi and medicinal plants, thus providing reference information for licorice growing for commercial medicinal purposes.

\section{Materials And Methods}




\subsection{Sample collection}

The roots and rhizosphere soils samples (soil depths were $0-20 \mathrm{~cm}, 20-40 \mathrm{~cm}$ and $40-60 \mathrm{~cm}$, respectively) of three medicinal Glycyrrhiza (Glycyrrhiza uralensis, Glycyrrhiza inflata and Glycyrrhiza glabra) were collected from August to September in 2019 from specimens growing at 3 distinct sites in 3 eco-regions in Xinjiang province, China; the geographical location of sampling points and soil physical and chemical properties are shown in Table 1. In addition, to ensure that the experiment was representative, we randomly selected three Glycyrrhiza plants in good growth condition from each geographical location according to the five-point sampling method, and all samples were cut with sterile scissors. The roots of each plant were divided into three sections: upper (0-20cm), middle $(20-40 \mathrm{~cm})$, and lower $(40-60 \mathrm{~cm})$, and the roots of each section are equally divided into two parts: one part was placed into a ziplocked bag for the determination of the bioactive compounds in the root, while the other part was placed into a sterile bag and quickly transported on a piece of ice to the laboratory in preparation for the microbe determination. All the samples were labeled by combination with letters and numbers, with the first letter representing the species (W, G and D: Glycyrrhiza uralensis, Glycyrrhiza glabra and Glycyrrhiza inflata, respectively), the second letter representing the root depth (1, 2 and 3: $0-20 \mathrm{~cm}, 20-40 \mathrm{~cm}, 40-60 \mathrm{~cm}$ ), and the third number representing the replicate number. For example, W.1.3 represents the third repetition of G/ycyrrhiza uralensis at $0-20 \mathrm{~cm}$.

Table 1

geographical sources and physicochemical properties of soil samples

\begin{tabular}{|c|c|c|c|}
\hline Sampling Site & Yiwu County, Xinjiang Province & Hami City, Xinjiang Province & Shihezi City, Xinjiang Province \\
\hline Plant Species & Glycyrrhiza uralensis & Glycyrrhiza inflata & Glycyrrhiza glabra \\
\hline Altitude (m) & 1372.8 & 806.1 & 340.2 \\
\hline Latitude and longitude & $43^{\circ} 33^{\prime} 58^{\prime \prime} \mathrm{N}, 94^{\circ} 81^{\prime} 86^{\prime \prime} \mathrm{E}$ & $42^{\circ} 84^{\prime} 48^{\prime \prime} \mathrm{N}, 93^{\circ} 54^{\prime} 80^{\prime \prime} \mathrm{E}$ & $44^{\circ} 45^{\prime} 18^{\prime \prime} \mathrm{N}, 86^{\circ} 06^{\prime} 39^{\prime \prime} \mathrm{E}$ \\
\hline Annual average temperature $\left({ }^{\circ} \mathrm{C}\right)$ & 5.5 & 9.8 & 8.1 \\
\hline Annual average precipitation $(\mathrm{mm})$ & 105.8 & 33.8 & 225 \\
\hline Total nitrogen $(\mathrm{g} / \mathrm{kg})$ & 0.832 & 0.762 & 0.693 \\
\hline Total phosphorus (g/kg) & 0.712 & 0.537 & 0.665 \\
\hline Total potassium(g/kg) & 19.743 & 21.864 & 20.771 \\
\hline $\mathrm{PH}$ & 8.534 & 8.45 & 8.831 \\
\hline Soil water content (\%) & 3.58 & 4.92 & 7.98 \\
\hline organic matter $(\mathrm{g} / \mathrm{kg})$ & 14.744 & 27.99 & 10.495 \\
\hline total salt $(\mathrm{g} / \mathrm{kg})$ & 1.033 & 5.697 & 4.894 \\
\hline nitrate nitrogen $(\mathrm{mg} / \mathrm{kg})$ & 7.592 & 14.2 & 3.552 \\
\hline ammonium nitrogen $(\mathrm{mg} / \mathrm{kg})$ & 6.021 & 4.869 & 3.326 \\
\hline Available phosphorus (mg/kg) & 3.699 & 9.677 & 5.292 \\
\hline Available potassium (mg/kg) & 81.208 & 180.032 & 273.093 \\
\hline
\end{tabular}

\subsection{Surface sterilization}

At the same time, to eliminate the interference of other microorganisms, the surface of roots was sterilized in the laboratory by first rinsing soil from the roots under running water followed by washing with sterile distilled water. The roots were then soaked in $75 \%$ alcohol for $30 \mathrm{~s}$ for surface disinfection, and then washed five times with sterile distilled water before soaking in $5 \%$ sodium hypochlorite for 5 min. Finally the roots were washed five times with sterile distilled water and air-dried under sterile conditions [36]. To confirm that the surface sterilization process was successful, the last rinse solution was inoculated onto a potato dextrose agar (PDA) plate and cultured at $28^{\circ} \mathrm{C}$ for $72 \mathrm{~h}$. No fungi growth confirmed that the surface sterilization was successful [37]. All root samples were labeled and immediately placed on ice and then stored at liquid nitrogen prior to total DNA extraction.

\subsection{Soil physicochemical}

Soil samples from the rhizosphere were air-dried and sieved through a 2-mm mesh for soil physicochemical properties analysis. The following soil physicochemical characteristics were analyzed according to the methods described by the Bao et al [38]: The content of organic matter (SOM) was determined by external heating with potassium dichromate. Soil pH (1:2.5 = soil: distilled water) was determined using a pH meter. Soil Water content (SWC) was determined by weighing. The total nitrogen (STN) content was determined using the perchloric acid-sulfuric acid digestion method. The total phosphorus (STP) content was determined by acid digestion (molybdenum-antimony colorimetry). The total potassium (STK) content was determined by acid digestion (atomic absorption spectrometry). The total salt (TS) content was determined by atomic absorption spectrometry. Nitrate nitrogen (SNN) and ammonium nitrogen (SAN) contents were analyzed using $0.01 \mathrm{M}$ calcium chloride extraction. The available phosphorus (SAP) content was determined by sodium bicarbonate extraction (molybdenum-antimony colorimetry). The available potassium (SAK) content was determined by ammonium acetate extract method (atomic absorption spectrometry).

\subsection{Determination of active components}


The root samples were dried at $60^{\circ} \mathrm{C}$ for $72 \mathrm{~h}$ to constant weight (it has been confirmed that glycyrrhizic acid (GIA) and liquiritin (LI) do not decompose at this temperature [39]. The dried root samples were ground to a powder with a pestle and mortar and passed through a 60 mesh sieve. An aliquot ( $0.2 \mathrm{~g})$ of powdered root sample was extracted with $71 \%$ chromatographic methanol in an ultrasonic bath $(250 \mathrm{~W}, 40 \mathrm{kHz})$ at room temperature. The extract was then centrifuged at $12,000 \mathrm{rpm}$ for 10 minutes and the supernatant was filtered (0.22- $\mu \mathrm{m}$ pore size) (Agilent, USA). The GIA and LI contents in the dried root samples $(0.2 \mathrm{~g})$ of the medicinal licorices were determined by high-performance liquid chromatography (HPLC, Agilent-1260 Infinity, USA) using an Agilent ZORBAX SB-C18 column $(150 \mathrm{~mm} \times 4.6 \mathrm{~mm}, 5 \mu \mathrm{m})$ with mobile phase (chromatographic methanol: ultra-pure water: $36 \%$ glacial acetic acid = 71:28:1) and mobile phase (acetonitrile:0.5\% glacial acetic acid $=1: 4$ ) respectively, and a gradient elution flow rate of $1.0 \mathrm{~mL} \cdot \mathrm{min}^{-1}$. GIA and LI were detected at $254 \mathrm{~nm}$ and $276 \mathrm{~nm}$, respectively. The injection volume was $5 \mu \mathrm{L}$ and the column temperature was $30^{\circ} \mathrm{C}$. The GIA and LI reference materials (CAS\#1405-86-3 and CAS \#551-15-5, respectively) were purchased from Solarbio and used for calibration purposes. The total flavonoid content (GTF) in medicinal licorices was determined by ultraviolet spectrophotometry at $334 \mathrm{~nm}$ with the liquiritin standard (CAS\#551-15-5) as the control.

\subsection{DNA extraction and library construction}

After immersion in liquid nitrogen, genomic DNA was extracted from the samples using the DNA Quick Plant System kit (Tiangen, China) according to the manufacturer's instructions. The purity and concentration of DNA were detected evaluated using a NanoDrop2000 (Thermo Fisher Scientific, USA). According to the concentration, each DNA sample was diluted a final concentration to $1 \mathrm{ng} / \mu \mathrm{L}$ with sterile distilled water for use as a DNA template.

The ITS (Internal Transcribed Spacer) rDNA genes of the ITS1 region were amplified using specific primers (ITS5-1737F 5'-GGAAGTAAAAGTCGTAACAAGG3'and ITS2-2043R 5'-GCTGCGTTCTTCATCGATGC-3') with barcodes [40]. PCR analyses were carried out with Phusion ${ }^{\circledR}$ High-Fidelity PCR Master Mix and GC Buffer (New England Biolabs) to ensure amplification efficiency and accuracy. PCR runs started at $95^{\circ} \mathrm{C}$ for 3 min, followed by 30 cycles of $95^{\circ} \mathrm{C}$ for $30 \mathrm{~s}$, $55^{\circ} \mathrm{C}$ for $30 \mathrm{~s}, 72{ }^{\circ} \mathrm{C}$ for $30 \mathrm{~s}$ and a final extension step at $72{ }^{\circ} \mathrm{C}$ for $5 \mathrm{~min}$.

The PCR product was mixed with the same volume of $1 \times$ TAE and then was detected by $2 \%$ agarose gel electrophoresis. The PCR product was purified from the target strip using a GeneJET Gel Extraction Kit (Thermo Scientific). The libraries were constructed using a TruSeq ${ }^{\circledR}$ DNA PCR-Free Sample Preparation Kit (Illumina, USA) according to the manufacturer's instructions, and index codes were added. The library quality was assessed on the Qubit @ 2.0 Fluorometer (Thermo Scientific) and Agilent Bioanalyzer 2100 system. Finally, Amplicon sequencing was performed using the Illumina HiSeq2500 platforms at the Beijing Compass Biotechnology Co., Ltd. (Beijing, China).

\subsection{Bioinformatics analysis and statistical analysis}

Single-end reads was assigned to samples using Cutadapt [41] software based on their unique barcode and truncated by cutting off the barcode and primer sequence. To avoid the influence of non-microbiota sequences (such as, chloroplast and mitochondrial sequences), the raw sequences were further filtered by Cutadapt software to remove non-microbiota taxa before subsequent analysis. Then raw tags were subjected to a strict quality controlled process using Cutadapt software to obtain high-quality clean reads. Clean reads were obtained by comparison with the reference database (Unite database) [42] using UCHIME algorithm to detect and remove chimeric sequences.

UPARSE software [43] (UPARSE v7.0.1001) was used to cluster the effective tags of all samples into the same operational taxonomic units (OTUs) with $\geq 97 \%$ identity, and taking the sequence with the highest frequency as the representative sequence of each OTU. The taxonomic information for each representative sequence was annotated using the Unite database, and multiple sequence alignment was performed using MUSCLE (Version 3.8.31) software to study the phylogenetic relationship of the representative sequences of OTUs among the 27 root samples. OTU abundance information was normalized using a standard sequence number corresponding to the sample with the least sequences $(54,262$ reads for sample D.2.1). Subsequent analysis of alpha diversity and beta diversity were performed based on this output normalized data. The raw sequence reads have been deposited in the NCBI Sequence Read Archive (SRA) with BioProject accession number PRJNA664554.

Using the FunGuild database based on the species information obtained from amplicon analysis, the ecological functions of existing species in the environment can be inquired.

Alpha Diversity analysis was used to study the complexity of species diversity in a sample through six indices (observed-species, Shannon, Simpson, Chao1, $\mathrm{ACE}$, and Good-coverage) [44]. All indices in the samples were calculated with QIIME (Version 1.7.0) and displayed with R software (Version 2.15.3).

Beta diversity analysis was used to evaluate differences in sample species complexity, which based on weighted Unifrac was calculated by QIIME software. The Un-weighted Pair-group Method with Arithmetic Mean (UPGMA) clustering analysis was conducted by QIIME software (Version 1.7.0). In addition, R software (Version 2.15.3) was also used to rarefaction curve generation, Wilcoxon rank sum test, Metastat statistical test, Spearman correlation analysis of heat maps and Distance-based Redundancy Analysis (db-RDA). Pearson correlation analysis was run among the bioactive compounds and the soil physicochemical properties. Two-way ANOVA was performed with SPSS 19.0 (IBM Inc., Armonk, USA), and displayed with GraphPad Prism 5.

\section{Results}

\subsection{Sequencing Results}

In the root of the three medicinal Glycyrrhiza species (Glycyrrhiza uralensis, Glycyrrhiza glabra, and Glycyrrhiza inflata), a total of 2,118,633 effective sequences were obtained after filtering out low-quality and short sequence reads. The sequencing results for each sample are listed in Supplementary table S1. The effective sequences were clustered into OTUs with $97 \%$ identity, and a total of 1,063 OTUs were obtained, among them, $91.53 \%$ of the effective sequences were assigned to the Kingdom level, $59.27 \%$ to the phylum level, $54.37 \%$ to the class level, $53.72 \%$ to the order level, $46.19 \%$ to the family level, 
$38.01 \%$ to the genus level, and $23.52 \%$ to the species level by the Illumina HiSeq (Fig. 1a). The rarefaction curves showed that the number of OTU in each sample increased gradually with quantity of sequence, thus confirming that the amount of sequencing data was adequate (Fig. $1 \mathrm{~b}$ ).

\subsection{Composition of fungal community in the root of medicinal liquorices}

According to the OTUs sequence and Unite database, 8 phyla, 23 classes, 53 orders, 102 families, 140 genera and 141 species were annotated. The endophytic fungal phyla with the greatest abundance from nine groups were enumerated in Fig. $2 \mathrm{a}$. Ascomycota dominated the observed sequences at the phylum level, representing $91.821 \%, 60.558 \%, 39.956 \%, 79.651 \%, 62.305 \%, 54.241 \%, 82.176 \%, 81.928 \%$ and $80.290 \%$ of the total number of species in D1, D2, D3, G1, G2, G3, W1, W2 and W3, respectively. In addition, Basidiomycota occupied a large part of the relative abundance in D2 (21.348\%), D3 (28.440\%), G2 (10.631\%), G3 (12.523\%), W2 (6.749\%) and W3 (5.110\%), respectively. Meanwhile, our results showed that the relative abundance of Ascomycota gradually decreased with the downward movement of root depths (Fig. 2b). For the difference analysis at the Phylum classification level, a MetaStat statistical test based on species abundance was constructed, and the results showed that the relative abundance of Ascomycota in Glycyrrhiza inflata significant difference distribution at different root depth (Fig. 2b). Specifically speaking, the relative abundance of Ascomycota at D1 sample (91.821\%) was significantly higher than D3 sample (39.956\%) (Fig. 2b).

In terms of genus, we listed the top 10 dominant fungal genera in each group in Fig. 2c: Fusarium was found to be the predominant genus in D1 (27.907\%), G1 (23.944\%), G2 (31.071\%), G3 (25.381\%), W1 (19.253\%) and W3 (18.215\%). Meanwhile, the abundance of Paraphoma was high in the D1, D3 and W3 samples, accounting for $27.738 \%, 23.937 \%$ and $13.980 \%$, respectively. Helminthosporium occupied a large part of the relative abundance in D1 (26.567\%), G1 (25.124\%), W1 (8.224\%) and W2 (17.408\%), respectively. Sarocladium occupied a large part of the relative abundance in D2 (3.326\%), G1 (16.547\%), G2 (17.243\%), G3 (21.897\%) and W1 (4.218\%), respectively, the abundance of Cladosporium was high in D2 (6.446\%), D3 (2.721\%) and W3 (15.174\%). Cadophora (13.200\%) and Psathyrella (10.917\%) were found to be the most dominant in D2 sample. Tomentella (14.472\%) was found to be the most dominant in D3 sample. Conocybe (12.068\%) was found to be the most dominant in G3 sample.

At the same time, details of the composition of the top 10 dominant fungi at other classification levels (Class, Older, Family, Species) were listed in Supplementary table S2. Specifically speaking, Sordariomycetes, Dothideomycetes and Agaricomycetes were dominate at the class taxonomic level; the dominant species at the order taxonomic level are Hypocreales, Pleosporales, Thelephorales; the dominant species at the family taxonomic level are Nectriaceae, Phaeosphaeriaceae, Massarinaceae; the dominant species at the species taxonomic level are Fusarium-solani, Paraphoma-radicina, Sarocladiumkiliense.

Based on the ITS amplicons analysis, we obtained the classification and abundance information of endophytic fungal community in root of medicinal licorices; we also pay attention to what role these species play in the ecosystem. The top 25 main ecological function of fungal species based on FunGuild analysis was shown in Fig. 3. Plant_Pathogen-Soil_Saprotroph-Wood_Saprotroph (32.072\%) was found to be the most dominant in G2 sample; Fungal_ Parasite-Plant_Pathogen (26.567\%) was found to be the most dominant in D1 sample; the abundance of Undefined_ Saprotroph was high in the G1, G2 and G3 samples, accounting for $21.271 \%, 28.330 \%$ and $35.555 \%$, respectively. Ectomycorrhizal $(21.187 \%)$ and Endophyte (13.208\%) were found to be the most dominant in D2 sample. However, a much higher proportion of unassigned ecological function existed in all groups.

\subsection{Effects of root depth and plant species on alpha diversity in the fungal community of roots}

The alpha diversity index of each group was shown in Table 2. Some indexes (Shannon and Chao1) respectively reflected the diversity and richness of microbial communities in samples, the greater the index, the higher the species diversity, the richer the distribution. The Shannon index of the W1 (4.910) sample was the highest. In contrast, that of the D1 (3.393) sample was the lowest. Moreover, we found that D1 had the lowest Chao1 (238.678) and ACE (253.105), while the D3 sample had the highest Chao1 (356.317) and ACE (355.694), respectively. Meanwhile, the results based on Wilcoxon rank sum test showed that the Shannon index was significantly different distribution between Glycyrrhiza uralensis and Glycyrrhiza inflata, especially 0-20cm at the root depth (Fig. 4a). Specifically, the Shannon index in W1 sample was significantly higher than D1 sample $(p<0.05)$. Furthermore, the Chao1 index in Glycyrrhiza inflata increased gradually with the downward movement of root depths, and based on Wilcoxon rank sum test showed that the Chao1 index in Glycyrrhiza inflate was significantly affected by root depth (Fig. 4b). Specifically, the Chao1 index in D3 sample was significantly higher than D1 sample ( $p<0.01)$; D2 sample was significantly higher than D1 sample $(p<0.05)$. 
Table 2

The alpha diversity indexes in each group

\begin{tabular}{|c|c|c|c|c|c|c|c|}
\hline \multirow[t]{2}{*}{ Sample name } & \multirow[t]{2}{*}{ Observed species } & \multicolumn{2}{|c|}{ Community diversity } & \multicolumn{2}{|c|}{ Community richness } & \multirow[t]{2}{*}{ Goods coverage } & \multirow[t]{2}{*}{ PD-whole tree } \\
\hline & & Shannon & Simpson & Chao1 & ACE & & \\
\hline D1 & 202.667 & 3.393 & 0.747 & 238.678 & 253.105 & 0.999 & 35.233 \\
\hline D2 & 302.333 & 4.678 & 0.907 & 327.516 & 338.868 & 0.999 & 63.772 \\
\hline D3 & 313.000 & 4.218 & 0.843 & 356.317 & 355.694 & 0.999 & 73.547 \\
\hline G1 & 254.000 & 3.897 & 0.829 & 282.714 & 292.674 & 0.999 & 51.440 \\
\hline $\mathrm{G} 2$ & 274.000 & 3.736 & 0.821 & 304.665 & 316.987 & 0.999 & 50.496 \\
\hline G3 & 282.000 & 4.060 & 0.826 & 320.275 & 329.183 & 0.999 & 48.561 \\
\hline W1 & 279.333 & 4.910 & 0.911 & 317.592 & 323.995 & 0.999 & 38.791 \\
\hline W2 & 269.667 & 4.246 & 0.863 & 303.285 & 312.849 & 0.999 & 40.653 \\
\hline W3 & 276.333 & 4.470 & 0.883 & 326.368 & 324.554 & 0.999 & 49.658 \\
\hline
\end{tabular}

\subsection{Effects of root depth and plant species on beta diversity in the fungal community of roots}

Beta diversity analysis was used to evaluate differences in microbial community composition among the samples. The Unweighted Pair-Group Method with Arithmetic (UPGMA) cluster analysis was performed to study similarity in the composition of endophytic fungal community among different samples, and the clustering results were integrated with species relative abundance at phyla taxon level in each group. As shown in Fig. $5 \mathrm{a}$, the results of UPGMA cluster tree based on Weighted Unifrac distances showed that samples from G3, G2, G1 and W1 were clustered together, and samples from W3, D2, W2 and D1 were clustered together (Fig. 5a). Meanwhile, for the difference analysis between the beta diversity, a Wilcoxon rank sum test based on Weighted Unifrac distances was constructed (Fig. 5b), and the results showed that there were significant differences in beta diversity between Glycyrrhiza uralensis and Glycyrrhiza inflata, which was consistent with UPGMA cluster tree. Specifically, there were significant differences in beta diversity between D1 and D2 samples $(P<0.05)$, D3 and W3 samples $(P<0.05)$, and D1 and W1 samples $(P<0.01)$ (Fig. 5b), which indicated there were significant differences in endophytic fungal community composition in roots of medicinal licorices between different species and different root depth.

\subsection{The relationship between endophytic fungal communities and the bioactive compounds and soil physicochemical properties}

The results of two-way ANOVA showed that the content of the bioactive compounds (glycyrrhizic acid (GIA), liquiritin (LI) and total flavonoid (GTF)) were not significantly affected by the interaction effect between root depth $(0-20 \mathrm{~cm}, 20-40 \mathrm{~cm}, 40-60 \mathrm{~cm})$ and plant species (Glycyrrhiza uralensis, Glycyrrhiza inflata, and Glycyrrhiza glabra) $(P>0.05)$ (Table 3). However, the content of $\mathrm{LI}$ was significantly affected by the main effect plant species $(P<0.05)($ Table 3 and Fig. 6). As shown in the Fig. 6, the contents of $\mathrm{LI}$ in root of Glycyrrhiza uralensis were significantly higher than those in $G / y c y r r h i z a$ inflate $(P<0.05)$, and the contents of LI in root of Glycyrrhiza uralensis were significantly higher than those in Glycyrrhiza glabra $(P<0.05)($ Fig. 6a).

Table 3

Effect of plant species and root depth on the bioactive compounds of licorice root

\begin{tabular}{|c|c|c|c|c|c|c|c|}
\hline Source & Dependent variable & Type III Sum of Squares & Degrees of freedom & Mean Square & $F$ & $p$ value & Partial Eta Squared \\
\hline \multirow[t]{3}{*}{ Plant species } & $\mathrm{GIA}$ & 2.939 & 2 & 1.469 & 3.554 & 0.050 & 0.283 \\
\hline & GTF & 0.028 & 2 & 0.014 & 0.201 & 0.820 & 0.022 \\
\hline & LI & 4.183 & 2 & 2.091 & 4.763 & 0.022 & 0.346 \\
\hline \multirow[t]{3}{*}{ root depth } & GIA & 0.344 & 2 & 0.172 & 0.416 & 0.666 & 0.044 \\
\hline & GTF & 0.022 & 2 & 0.011 & 0.158 & 0.855 & 0.017 \\
\hline & $\mathrm{LI}$ & 1.466 & 2 & 0.733 & 1.670 & 0.216 & 0.156 \\
\hline \multirow[t]{3}{*}{ Species*root depth } & $\mathrm{GIA}$ & 0.268 & 4 & 0.067 & 0.162 & 0.955 & 0.035 \\
\hline & GTF & 0.066 & 4 & 0.016 & 0.233 & 0.916 & 0.049 \\
\hline & $\mathrm{LI}$ & 0.085 & 4 & 0.021 & 0.049 & 0.995 & 0.011 \\
\hline
\end{tabular}


In addition, Pearson correlation analysis showed that the content of bioactive compounds was significantly correlated with soil physicochemical properties (Supplementary table S3). GIA content in root had a very significant positive correlation with available potassium (SAK) and soil water content (SWC) ( $r>0 ; P$ $<0.05)$, but LI content in root had a very significant negative correlation with SAK and total salt (TS) content $(r<0 ; P<0.05)$.

Furthermore, spearman correlation analysis showed that the content of LI was significantly positive correlated with alpha diversity index $(r>0, P<0.05)$ (Fig. 7). As shown in Fig. 7, the content of LI had a very significant positive correlation with Shannon index, Simpson index and Chao1 index ( $P<0.05)$, which indicated that the content of LI was accountable for the diversity of endophytic fungal community in this study.

Meanwhile, spearman correlation analysis showed that there was a significant relationship between dominant fungi phylum and bioactive compounds and soil physicochemical properties (Table 4). Specifically, Ascomycota showed a very significant negative correlation with RWC ( $r<0, P<0.01)$; Basidiomycota showed a very significant positive correlation with RWC $(r>0 ; P<0.01)$; Olpidiomycota showed a significant positive correlation with GIA $(r>0 ; P<0.05)$; Mortierellomycota showed a significant positive correlation with STK, SWC and SAK $(r>0 ; P<0.05)$; Mucoromycota showed a very significant positive correlation with SOM $(r>0 ; P<0.01)$, but a significant negative correlation with STP $(r<0, P<0.01)$; Rozellomycota showed a significant positive correlation with SOM, STK and RWC $(r>0 ; P<0.05)$.

Table 4

Spearman correlation analyses testing the relationship between the relative abundance of dominant fungi phyla and bioactive compounds and soil physicoch properties.

\begin{tabular}{|c|c|c|c|c|c|c|c|c|}
\hline & Ascomycota & Basidiomycota & Glomeromycota & Olpidiomycota & Mortierellomycota & Mucoromycota & Rozellomycota & Neocallimastigor \\
\hline SOM & 0.033 & 0.243 & -0.184 & -0.090 & -0.028 & $0.579 * \star$ & $0.425^{\star}$ & 0.013 \\
\hline STN & 0.223 & 0.051 & -0.105 & 0.233 & -0.109 & -0.078 & -0.109 & 0.304 \\
\hline STP & 0.143 & -0.173 & -0.145 & 0.196 & -0.202 & $-0.544^{\star *}$ & -0.353 & 0.029 \\
\hline STK & -0.158 & 0.146 & -0.172 & -0.359 & $0.389 *$ & 0.295 & $0.409 *$ & -0.286 \\
\hline SNN & 0.069 & 0.132 & -0.188 & -0.003 & -0.113 & 0.330 & 0.262 & 0.161 \\
\hline SAN & 0.267 & -0.085 & -0.329 & -0.272 & -0.260 & -0.003 & 0.153 & -0.121 \\
\hline SAP & -0.231 & 0.299 & 0.078 & 0.119 & -0.030 & 0.021 & 0.167 & 0.163 \\
\hline SAK & -0.249 & 0.083 & 0.334 & 0.245 & $0.384^{\star}$ & -0.176 & -0.024 & 0.101 \\
\hline TS & -0.190 & 0.155 & 0.069 & -0.010 & 0.263 & -0.012 & 0.113 & 0.108 \\
\hline $\mathrm{PH}$ & -0.075 & 0.268 & -0.051 & 0.042 & -0.024 & 0.260 & -0.034 & 0.207 \\
\hline SWC & -0.237 & 0.047 & 0.347 & 0.090 & $0.504^{\star *}$ & 0.066 & 0.010 & 0.066 \\
\hline RWC & $-0.51^{\star \star}$ & $0.492^{\star \star}$ & 0.132 & -0.374 & 0.229 & 0.320 & $0.459 *$ & -0.008 \\
\hline GIA & 0.033 & -0.209 & 0.213 & $0.416^{*}$ & 0.060 & -0.056 & -0.128 & 0.093 \\
\hline GTF & -0.358 & 0.157 & 0.361 & 0.203 & 0.175 & -0.081 & -0.303 & -0.242 \\
\hline LI & -0.133 & 0.076 & 0.003 & 0.169 & -0.037 & -0.209 & 0.008 & 0.026 \\
\hline
\end{tabular}

The values are the correlation coefficients $(r)$. ** means $\mathrm{P}<0.01$; * means $\mathrm{P}<0.05$.

As shown in Fig. 8, there was a significant relationship between the dominant fungi genus and bioactive compounds and soil physicochemical properties. Specifically, Fusarium showed a significant positive correlation with $\mathrm{LI}$ content $(P<0.05)$; Paraphoma showed a significant positive correlation with SAN $(P<$ 0.05), but a significant negative correlation with SAK, TS and SWC $(P<0.05)$; Helminthosporium showed a significant positive correlation with $\mathrm{PH}(P<0.05)$; Sarocladium showed a significant negative correlation with SOM, STN and SNN $(P<0.05)$; Conocybe showed a significant positive correlation with SWC, but a significant negative correlation with SAN $(P<0.05)$.

Distance-based redundancy analysis (db-RDA) based on the Bray-Curtis distance showed that the bioactive compounds and soil physicochemical had significant effects on the differences of endophytic fungal community (Fig. 9). The differential distribution of endophytic fungal community was mainly restricted in the first and second ordination axes, and the first ordination axis, the second ordination axis were explained $16.23 \%, 13.89 \%$ of the total variability, respectively (Fig. 9). Specifically, among the soil environment factors, SAK content was identified as the factor that most significantly affects the differences of endophytic fungal community $\left(r^{2}=0.329, P<0.01\right)$, followed by SAN $(P<0.05)$. Among the root factors, the RWC was explained the difference of endophytic fungal communities in roots to the greatest extent $\left(r^{2}=0.247, P<0.05\right)$, followed by LI content $(P<0.05)$ (Fig. 9, Table 5). According to results of the db-RDA analysis, the SAN, SAK, RWC, and LI content were the major factors contributing to the variations in the overall structure of endophytic fungal community in this study. 
Table 5

Results for db-RDA testing effects of soil physicochemical properties and bioactive compounds on the composition and distribution of fungal community in licorice root.

\begin{tabular}{|lll|}
\hline & \multicolumn{1}{|c|}{$\operatorname{Pr}(\boldsymbol{r})$} \\
\hline SOM & 0.202 & 0.071 \\
\hline STN & 0.089 & 0.329 \\
\hline STP & 0.099 & 0.291 \\
\hline STK & 0.135 & 0.174 \\
\hline SNN & 0.175 & 0.100 \\
\hline SAN & 0.231 & 0.044 \\
\hline SAP & 0.070 & 0.423 \\
\hline SAK & 0.329 & 0.008 \\
\hline TS & 0.099 & 0.271 \\
\hline PH & 0.053 & 0.524 \\
\hline SWC & 0.121 & 0.220 \\
\hline RWC & 0.247 & 0.027 \\
\hline GIA & 0.026 & 0.730 \\
\hline GTF & 0.038 & 0.634 \\
\hline LI & 0.243 & 0.034 \\
\hline Description: ${ }^{2}$ is the determinant coefficients of the distribution of the fungal community by environmental factors. \\
\hline
\end{tabular}

\section{Discussion}

In this study, we investigated the composition and diversity of endophytic fungal communities in different root depth $(0-20 \mathrm{~cm}, 20-40 \mathrm{~cm}$ and $40-60 \mathrm{~cm})$ of three medicinal licorices (Glycyrrhiza uralensis, Glycyrrhiza glabra, and Glycyrrhiza inflata) using high-throughput sequencing technology, which provides a large amount of data with more accuracy than that obtained in previous studies using traditional technology [45-47]. We obtained the composition of endophytic fungal communities at different taxonomic levels (phylum, class, older, family, genus and species) by high-throughput sequencing (Fig. 2a, Fig. 2c and Supplementary table S2). The results showed that there was a specific microbiome in 27 samples of tree medicinal licorices, and the relative abundance of endophytic fungi was correlated with the host plant species and root depth. For example, Ascomycota was the dominant phylum in all samples, followed by Basidiomycota, which result consistent with previous studies $[48,49]$. The phylum Ascomycota, as the largest phylum of fungi, has diverse populations and plays an important role in genetics [50], ecology [51] and phylogeny [52]. Such as, the Ascomycota produce large numbers of spores through both asexual and sexual reproduction. Asci can act as small water cannon, spraying spores into the air. Dispersal process of ascospores, spores is important for dissemination of many fungal plant diseases and for the dispersal of many saprophytic fungi [53].

Moreover, our results showed that the relative abundance of Ascomycota gradually decreased with the downward movement of root depths (Fig. 2b), which was consistent with the results of Ko, Daegeun et al [54]. On this basis, we found that the relative abundance of Ascomycota in Glycyrrhiza inflata had a significant difference at different root depth, but Glycyrrhiza uralensis and Glycyrrhiza glabra were not significant difference, indicating that some endophytes may preferentially proliferate in a certain ecological region and play different ecological roles from other endophytes. Overall, in addition to soil depth, the relative abundance of endophytes was also related to the genotype of the host plant species. This was consistent with the results of host genotype and soil conditions on ectomycorrhizal community of poplar clones by Karliński, Leszek et al. [55].

Alpha Diversity and Beta Diversity analysis of endophytic fungal community showed significant differences in root depths $(0-20 \mathrm{~cm}, 20-40 \mathrm{~cm}$ and $40-60 \mathrm{~cm})$ between Glycyrrhiza uralensis and Glycyrrhiza inflata (Fig. 4, Fig. 5), which indicated that both genotype and ecological region of host plants contributed to the differences of endophytic fungal community. Meanwhile, numerous studies [56] have shown that the adaptation of endophytic fungal community largely depends on the adaptation of host plants to the ecological environment, which indicated that host plants largely determine the colonization and distribution of endophytic fungal community. The relationship between fungus and host plant were also often considered as a flexible interaction, with orientations determined by subtle differences in the expression of fungal genes in response to the host, or conversely, by the host's recognition and response to the fungus. Thus, slight genetic differences in the two genomes control the symbiosis [57].

Furthermore, our results showed that the root depth had a significant effect on the richness and composition of endophytic fungal community (Fig. 4b, Fig. 5a and Fig. 5b), which indicated that different ecological types of endophytic fungi may represent certain ecological regions (different root depth), these should become an important consideration factor that endophytic fungi inoculation. We speculated that this is related to root respiration and soil $\mathrm{C}$ content. On the one hand, root respiration, accounts for $60 \%$ of total soil respiration, can regulates the metabolism of roots and related microorganisms, and is an important part of terrestrial carbon budget [58]; on the other hand, the content of $\mathrm{C}$ in unstable soil varies greatly between different soil depths [59]. Moreover, Noah Fierer et al. [60] demonstrated that the vertical distribution of the specific microbial species was largely related to the decrease in carbon availability with soil depth. 
However, one weakness in this study was that the samples of three glycyrrhiza species were collected from areas which differed in geographical environment. Since it is rare to find three glycyrrhiza species in the same habitat, to a certain extent, the soil physicochemical properties can represent the environmental factors in which three glycyrrhiza species were growing. Therefore, in this study, in addition to root factor, also included the effects of the soil factors.

Numerous studies $[61,62]$ showed the accumulation of bioactive compounds in medicinal licorice roots is affected by various factors. In this study, the content of LI were more affected by main effect plant species than main effect root depth (Table 3), among them, the content of LI in root of Glycyrrhiza uralensis were significantly higher than those in Glycyrrhiza inflata and Glycyrrhiza glabra (Fig. 6a), which is consistent with the results of Zhang et al. [63]. We speculate that this is related to the expressions of some functional genes that are closely associated with the content of bioactive compounds including glycyrrhizic acid and liquiritin in root of licorice species. Some studies [64-66] have shown that key functional genes, such as chalcone synthase gene, 3Hydroxy-3-methylglutary CoA reductase (HMGR) and squalene synthase (SQS), are involved in transcriptional level regulation process in glycyrrhizic acid and liquiritin biosynthesis. Although further studies are required to characterize the expression of functional genes of bioactive compounds, this study provides a theoretical basis for the development of strategies to expand the Glycyrrhiza uralensis cultivation. On the other hand, the content of bioactive compounds is the result of the interaction between plants and their growing environment, therefore, the accumulation of bioactive compounds in root is influenced by the ecological environment of its. In this study, GIA, GTF and LI content had a positive correlation with soil total nitrogen (STN) ( $r>0$ ), indicating that soil nutrients can promote the accumulation of bioactive compounds, but not all soil nutrients, such as soil total potassium (STK), have such a function. Although potassium can be involved in many enzyme activation systems in plants and improve plant stress resistance [67], the content of GIA, GTF and LI were negatively correlated with STK $(r<0)$ in this study, which is consistent with the results of Liu et al [68]. In addition, soil available potassium (SAK) had a significant positive correlation with GIA, but had a significant negative correlation with LI (Supplementary table S3), indicating that the utilization mechanism of soil nutrients by bioactive compounds is completely different. Although the mechanism by which available potassium regulate bioactive compounds is still unclear, this discovery may form the basis of further in-depth research. In general, these soil factors exhibit habitat specific characteristics are related to the regulation of bioactive compounds in root of licorice.

In recent years, a growing number of studies [69-71] have demonstrated that the dynamics of the microflora is driven to a large extent by environmental factors including soil characteristics ( $\mathrm{pH}$, nitrogen, phosphorus and potassium) and climate condition (rainfall and temperature). Consistent with these reports, our results showed that LI, RWC, SAN and SAK content were the major factors contributing to the variations in the overall structure of endophytic fungal community (Fig. 9 and Table 5). In addition, we found that the content of LI in root had a very significant positive correlation with diversity of endophytic fungal community (Shannon and Simpson index) $(P<0.05)$ (Fig. 7). Liquiritin (LI), the main bioactive compounds of flavonoids, is one of the material basis for clinical efficacy and an important index of the quality of medicinal licorices. Flavonoids can be specifically induced by symbiotic fungus to respond to purified signaling molecules from these organisms when the fungus colonizes. Chen et al. [72] demonstrated that with inoculation of fungi Glomus mosseae, Glycyrrhiza uralensis plants significantly increased stem and root biomass and liquiritin content in the main root.

Meanwhile, our results showed that soil physicochemical and bioactive compounds had a significant effect on composition of endophytic fungal communities (such as phylum and genus) (Fig. 8 and Table 4), which showed that there is an interaction among endophytic fungal community, root and soil factor. This suggests that we may be able to alter the fungal composition by altering soil factors [73], thereby promoting the accumulation of bioactive compounds in plants [74]. In the case of medicinal licorices, Wei Xie et al. [75] shown that P addition and arbuscular mycorrhizal (AM) inoculation could improve plant growth and facilitated glycyrrhizic acid and liquiritin accumulation in Glycyrrhiza uralensis. Meanwhile, Y. Orujei et al. [76] also shown that two species of arbuscular mycorrhizal fungi (AMF) were successful inoculation, the increase in the growth rate and the accumulation of bioactive compounds in licorice roots ( Glycyrrhiza glabra) were observed compared to control. In general, this study provided useful an information for the development of strategies to improve the production and quality of medicinal licorices, although further studies are required to characterize the functions of these endophytic fungi.

\section{Conclusions}

In this study, numerous endophytic fungal communities were detected in roots of medicinal licorices based on high-throughput sequencing. Furthermore, we identified significant differences in the relative abundance of Ascomycota among root depth. Furthermore, the alpha diversity analysis and beta diversity analysis showed that the endophytic fungal community structure and composition differed among the species and root depth in medicinal licorices. Moreover, the SAN, SAK, RWC, and LI content were the major factors contributing to the variations in the overall structure of endophytic fungal community in this study. This study clarified the ecological role of non-biological factor (soil and root) in the endophytic fungal community of medicinal licorices, which may provide theoretical basis for the synthesis of bioactive compounds and rational utilization of medicinal plants in production practice.

\section{Abbreviations}

PDA

potato dextrose agar

SOM

organic matter

STN

total nitrogen

STP

total phosphorus

STK

total potassium

TS 
total salt

SNN

nitrate nitrogen

SAN

ammonium nitrogen

SAP

available phosphorus

SAK

available potassium

SWC

Soil water content

HPLC

high-performance liquid chromatography

GIA

glycyrrhizic acid

LI

liquiritin

GTF

total flavonoid

OTUs

operational taxonomic units

SRA

Sequence Read Archive

UPGMA

Un-weighted Pair-group Method with Arithmetic Mean

Db-RDA

Distance-based Redundancy Analysis

\section{Declarations}

\section{Ethics approval and consent to participate}

Not applicable

\section{Adherence to national and international regulations}

Field studies were conducted in accordance with local legislation.

\section{Consent for publication}

Not applicable

\section{Availability of data and material}

All data generated or analysed during this study are included in this published article and its supplementary information files.

\section{Competing interests}

The authors declare that they have no competing interests.

\section{Funding}

This study was financially supported by the National Natural Science Foundation of China (41561010 and 31560177).

\section{Authors' contributions}

L.Z. and .H.D. designed the experiment. H.D. performed methodology, data analysis, investigated and was a major contributor in writing the manuscript. G. Li., Y. Mu, X. Lv. and Z. W. collected the samples and modified the manuscript. All authors read and approved the final manuscript. 


\section{Acknowledgements}

In this study, we would like to thank professor L.Z for hers guidance, all the authors for their joint efforts. We also would like to thank many graduate students and staff who directed the collection of soil samples that were not listed as co-authors.

\section{References}

1. Dao-Yuan Z, Hong-Ling W (2005) Preliminary Study on the Growth Pattern of Several Clonal Plants in Desert Zones of Xinjiang. Arid Zone Research 22: 219-224.

2. Rizzato G, Scalabrin E, Radaelli M, Capodaglio G, Piccolo O (2017) A new exploration of licorice metabolome. Food Chemistry 221:959-968.

3. Wang L, Yang R, Yuan B, Liu Y, Liu C (2015) The antiviral and antimicrobial activities of licorice, a widely-used Chinese herb. Acta Pharmaceutica Sinica B 5:310-315.

4. Tianshui Niu JY, Long Zhang, Xiao Cheng, Kai Li, Gang Zhou (2009) Research Advances on Anticancer Effect of Licorice. Current Bioactive Compounds 5: 234-242.

5. Li-Ping L, Cui-Ai R, Hong-Yan Z (2010) Research Progress on Immunomodulatory Effects of Glycyrrhizin. chinese journal of experimental traditional medical formulae 6: 272-276.

6. Schr?Felbauer B, Raffetseder J, Hauner M, Wolkerstorfer A, Ernst W, Szolar OHJ (2009) Glycyrrhizin, the main active compound in liquorice, attenuates pro-inflammatory responses by interfering with membrane-dependent receptor signalling. Biochemical Journal 421:473-482.

7. Baba M, Shigeta S (1987) Antiviral activity of glycyrrhizin against varicella-zoster virus in vitro. Antiviral Research 7:99-107.

8. Crance JM, Scaramozzino N, Jouan A, Garin D (2003) Interferon, ribavirin, 6-azauridine and glycyrrhizin: antiviral compounds active against pathogenic flaviviruses. Antiviral Res 58:73-79.

9. Yina L, A EG, A YZ, A ZS, Wang B (2018) Chemical Profile and Anti-inflammatory Activity of Total Flavonoids from Glycyrrhiza Uralensis Fisch. Iranian Journal of Pharmaceutical Research 17:726-734.

10. Weidner S, Kordala E, Brosowska-Arendt W, Karamac M, Kosinska A, Amarowicz R (2009) Phenolic compounds and properties of antioxidants in grapevine roots (Vitis vinifera L.) under drought stress followed by recovery. Acta Societatis Botanicorum Poloniae 78:279-286.

11. Antolak H, Czyzowska A, Kregiel D (2016) Antibacterial and Antiadhesive Activities of Extracts from Edible Plants against Soft Drink Spoilage by Asaia spp. Journal of food protection 80:25-34.

12. Ncube B, Finnie JF, Van Staden J (2012) Quality from the field: The impact of environmental factors as quality determinants in medicinal plants. South African Journal of Botany 82:11-20.

13. Han T, Jia M, Lu B, Qin L (2013) Relationships between endophytic fungi and their hosts: impacts on drug quality of medicinal plants. Planta Medica 79: 1256.

14. S., H., Faeth (2002) Fungal Endophytes: Common Host Plant Symbionts but Uncommon Mutualists. Integrative and Comparative Biology $42: 360-368$.

15. Huang WY, Cai YZ, Xing J, Corke H, Sun M (2007) A potential antioxidant resource: Endophytic fungi from medicinal plants. Economic Botany 61:14-30.

16. Jia M, Chen L, Xin H-L, Zheng C-J, Rahman K, Han T, Qin L-P (2016) A friendly relationship between endophytic fungi and medicinal plants: a systematic review. Frontiers in microbiology 7:906.

17. Vaz ABM, Mota RC, Bomfim MRQ, Vieira MLA, Zani CL, Rosa CA, Rosa LH (2009) Antimicrobial activity of endophytic fungi associated with Orchidaceae in Brazil. Canadian Journal of Microbiology 55: 1381-1391.

18. Hernawati H, Wiyono S, Santoso S (2011) Leaf endophytic fungi of chili (Capsicum annuum) and their role in the protection against Aphis gossypii (Homoptera: Aphididae). Biodiversitas 12: 187-191.

19. Radi? T, Likar M, Han?Evi? K, Bogdanovi? I, Paskovi? I (2014) Occurrence of root endophytic fungi in organic versus conventional vineyards on the Croatian coast. Agriculture Ecosystems \& Environment 192:115-121.

20. Singh G, Mukerji KG (2006) Root Exudates as Determinant of Rhizospheric Microbial Biodiversity. Microbial Activity in the Rhizoshere 7: $39-53$.

21. Jihui Z (1999) Studies on the Plant Hormones Produced by 5 Species of Endophytic Fungi Isolated from Medicinal Plants (Orchidacea). ACTA ACADEMIAE MEDICINAE SINICAE 21: 460-465.

22. Zhao J, Shan T, Mou Y, Zhou L (2011) Plant-Derived Bioactive Compounds Produced by Endophytic Fungi. Mini Reviews in Medicinal Chemistry 11: 159168.

23. Ratnaweera PB, Silva EDD, Williams DE, Andersen RJ (2015) Antimicrobial activities of endophytic fungi obtained from the arid zone invasive plant Opuntia dillenii and the isolation of equisetin, from endophytic Fusarium sp. BMC Complementary and Alternative Medicine 15:220.

24. Farhana, Alam, Ripa, Wei-Dong, Cao, Shuai, Tong, Jian-Guang, Sun (2019) Assessment of Plant Growth Promoting and Abiotic Stress Tolerance Properties of Wheat Endophytic Fungi. Biomed Research International 2019.

25. Firáková S, ?turdíková M, Mú?ková M (2007) Bioactive secondary metabolites produced by microorganisms associated with plants. Biologia 62:251-257.

26. Saikkonen K, Faeth SH, Helander M, Sullivan TJ (1998) FUNGAL ENDOPHYTES: A Continuum of Interactions with Host Plants. Annual Review of Ecology \& Systematics 29:319-343.

27. Pimentel IC, Glienke-Blanco C, Gabardo J, Stuart RM, Azevedo JL (2006) Identification and colonization of endophytic fungi from soybean (Glycine max (L.) Merril) under different environmental conditions. Brazilian Archives of Biology and Technology 49: 705-711. 
28. Liu W, Zhou Z, Liu Y, Hu X, Guo Y, Li J (2017) Application of High-Throughput Internal Transcribed Spacer rRNA Metagenomics Analysis in Deciphering Endophytic Fungi Diversity of Dendrobium Officinale. Journal of Biobased Materials and Bioenergy 11:106-118.

29. Coutlyne MM, Thierry R, Ajay K, Cornelius BC, Njie AC (2018) Phylogenetic analysis and diversity of novel endophytic fungi isolated from medicinal plant Sceletium tortuosum. Phytochemistry Letters 27:36-43.

30. Taylor DL, Hollingsworth TN, Mfarland JW, Lennon NJ, Nusbaum C, Ruess RW (2014) A first comprehensive census of fungi in soil reveals both hyperdiversity and fine-scale niche partitioning. Ecological Monographs 84: 3-20.

31. Blackwell, M ( 2011) The Fungi: 1, 2, 3 ... 5.1 million species?, 98:426-438.

32. Kivlin SN, Lynn JS, Kazenel MR, Beals KK, Rudgers JA (2017) Biogeography of plant $\rrbracket s s o c i a t e d$ fungal symbionts in mountain ecosystems: A meta nalysis. Diversity and Distributions 23:1067-1077.

33. Berg G (2009) Plant-microbe interactions promoting plant growth and health: perspectives for controlled use of microorganisms in agriculture. Applied Microbiology \& Biotechnology 84:11-18.

34. Kathrin, Blumenstein, David, Macaya-Sanz, Juan, A., Martín, Benedicte, R., Albrectsen (2015) Phenotype MicroArrays as a complementary tool to next generation sequencing for characterization of tree endophytes. Frontiers in Microbiology 6:1033.

35. Karliński L, Rudawska M, Kieliszewska-Rokicka B, Leski T (2010) Relationship between genotype and soil environment during colonization of poplar roots by mycorrhizal and endophytic fungi. Mycorrhiza 20:315-324.

36. Saude C, Hurtado-Gonzales OP, Lamour KH, Hausbeck MK (2008) Occurrence and characterization of a Phytophthora sp. pathogenic to asparagus (Asparagus officinalis) in Michigan. Phytopathology 98:1075-1083.

37. Jin-Long C, Vinod V, Gang Z (2018) Partitioning of Fungal Endophyte Assemblages in Root-Parasitic Plant Cynomorium songaricum and Its Host Nitraria tangutorum. Frontiers in Microbiology 9:666.

38. Bao S (2008) Soil Agro-chemistrical Analysis. China Agriculture Press 22-196.

39. Jing Q, Zuliang L, Yanpeng L, Guangxi R, Chunsheng L, Xiaojun M (2017) Effect of Abscisic Acid on Accumulation of Five Active Components in Root of Glycyrrhiza uralensis. Molecules 22:1982.

40. Berry D, Ben Mahfoudh K, Wagner M, Loy A (2012) Barcoded Primers Used in Multiplex Amplicon Pyrosequencing Bias Amplification. Applied \& Environmental Microbiology 78:612-612.

41. Liu, Zhenshan, Wei, Gehong, Yang, Jun, Chen, Weimin, Lin, Yanbing (2016) Bacterial communities in oil contaminated soils: Biogeography and cooccurrence patterns. Soil Biology \& Biochemistry 98: 64-73.

42. Brian J, Haas, Dirk, Gevers, Ashlee M, Earl, Mike, Feldgarden, Doyle V, Ward (2011) Chimeric 16S rRNA sequence formation and detection in Sanger and 454-pyrosequenced PCR amplicons. Genome research 21: 494-504.

43. Martin M (2011) Cutadapt removes adapter sequences from high-throughput sequencing reads. Embnet Journal, 17: 10-12.

44. Li B, Zhang X, Guo F, Wu W, Zhang T (2013) Characterization of tetracycline resistant bacterial community in saline activated sludge using batch stress incubation with high-throughput sequencing analysis. Water Research 47:4207-4216.

45. Weifang T, Yuanlei H, Zhu (2010) Isolation and Identification of Endophytical Fungus in Licorice Collected from Xinjiang Area. Biotechnology Bulletin 20:149-153.

46. Jiang-Tao BI, Xiao-Xia W, Wei-Min C, Jing W, Da-Han HE (2013) Isolation of endophytic fungi from medicinal plant Glycyrrhiza uralensis and its microbial inhibition activity. Pratacultural ence 30:357-364.

47. Palak, Arora, Zahoor, Wani, Tanveer, Ahmad, Phalisteen, Sultan, Suphla (2019) Community structure, spatial distribution, diversity and functional characterization of culturable endophytic fungi associated with Glycyrrhiza glabra L. Fungal Biology 123: 373-383.

48. Stephenson SL, Tsui C, Rollins AW (2013) Methods for Sampling and Analyzing Wetland Fungi: Springer Netherlands 93-121.

49. Tan X, Zhou Y, Zhou X, Xia X, Wei Y, He L, Tang H, Yu L (2018) Diversity and bioactive potential of culturable fungal endophytes of Dysosma versipellis; a rare medicinal plant endemic to China. Scientific Reports 8: 1-9.

50. Wallen RM, Perlin MH (2018) An overview of the function and maintenance of sexual reproduction in dikaryotic fungi. Frontiers in microbiology 9:503.

51. Belnap J, Lange OL (2005) Lichens and microfungi in biological soil crusts: community structure, physiology, and ecological functions. MYCOLOGY SERIES 23:117.

52. Schoch CL, Sung G-H, López-Giráldez F, Townsend JP, Miadlikowska J, Hofstetter V, Robbertse B, Matheny PB, Kauff F, Wang Z (2009) The Ascomycota tree of life: a phylum-wide phylogeny clarifies the origin and evolution of fundamental reproductive and ecological traits. Systematic biology 58:224-239.

53. Trail F (2007) Fungal cannons: explosive spore discharge in the Ascomycota. FEMS microbiology letters 276:12-18.

54. Ko D (2015) Variations in bacterial and fungal community composition along the soil depth profiles determined by pyrosequencing. AGUFM 2015: B11J0579.

55. Karliński L, Rudawska M, Leski T (2013) The influence of host genotype and soil conditions on ectomycorrhizal community of poplar clones. European Journal of Soil Biology 58:51-58.

56. Saikkonen K, Wäli P, Helander M, Faeth SH (2004) Evolution of endophyte-plant symbioses. Trends in plant science 9:275-280.

57. Moricca S, Ragazzi A (2008) Fungal endophytes in Mediterranean oak forests: a lesson from Discula quercina. Phytopathology 98:380-386.

58. Pregitzer KS, Laskowski MJ, Burton AJ, Lessard VC, Zak DR (1998) Variation in sugar maple root respiration with root diameter and soil depth. Tree Physiology 18:665-670.

Page $12 / 19$ 
59. de Graaff M-A, Jastrow JD, Gillette S, Johns A, Wullschleger SD (2014) Differential priming of soil carbon driven by soil depth and root impacts on carbon availability. Soil Biology \& Biochemistry 69:147-156.

60. Fierer N, Schimel JP, Holden PA (2003) Variations in microbial community composition through two soil depth profiles. Soil Biology \& Biochemistry 35:167-176.

61. Li Y, Wu H (2018) The Research Progress of the Correlation Between Growth Development and Dynamic Accumulation of the Effective Components in Medicinal Plants. Chinese Bulletin of Botany 3: 293-304.

62. Da-Cheng, Hao, Pei-Gen, Xiao (2015) Genomics and Evolution in Traditional Medicinal Plants: Road to a Healthier Life. Evolutionary Bioinformatics Online 11: EBO. S31326.

63. Zhang Y, Yang Y, Gong H, Zhu H (2018) A systematic review of the comparison of three medicinal licorices, based on differences of the types and contents about their bioactive components. Journal of Chemical Biology and Pharmaceutical Chemistry 1:1-6.

64. Winkel-Shirley B (2002) Biosynthesis of flavonoids and effects of stress. Current Opinion in Plant Biology 5:218-223.

65. Mochida K, Sakurai T, Seki H, Yoshida T, Takahagi K, Sawai S, Uchiyama H, Muranaka T, Saito K (2017) Draft genome assembly and annotation of Glycyrrhiza uralensis, a medicinal legume. The Plant Journal 89: 181-194.

66. Li J, Liu S, Wang J, Li J, Li J, Gao W (2017) Gene expression of glycyrrhizin acid and accumulation of endogenous signaling molecule in Glycyrrhiza uralensis Fisch adventitious roots after Saccharomyces cerevisiae and Meyerozyma guilliermondii applications. Biotechnology and Applied Biochemistry 64: 700-711.

67. Wang, Wu, WH (2017) Regulation of potassium transport and signaling in plants. Current Opinion in Plant Biology 39: 123-128.

68. Liu Y, Li Y, Luo W, Liu S, Chen W, Chen C, Jiao S, Wei G (2020) Soil potassium is correlated with root secondary metabolites and root-associated core bacteria in licorice of different ages. Plant and Soil 2020:1-19.

69. Edwards J, Johnson C, Santos-Medellín C, Lurie E, Sundaresan V (2015) Structure, variation, and assembly of the root-associated microbiomes of rice. Proceedings of the National Academy of ences of the United States of America 112: E911-E920.

70. Nuccio EE, Anderson-Furgeson J, Estera KY, Pett-Ridge J, De Valpine P, Brodie EL, Firestone MK (2016) Climate and edaphic controllers influence rhizosphere community assembly for a wild annual grass. Ecology 97:1307-1318.

71. Stegen JC, Lin X, Konopka AE, Fredrickson JK (2012) Stochastic and deterministic assembly processes in subsurface microbial communities. Isme Journal 6:1653-1664.

72. Meilan, Chen, Guang, Yang, Ye, Sheng, Pengying, Li, Hongyan, Qiu (2017) Glomus mosseae Inoculation Improves the Root System Architecture, Photosynthetic Efficiency and Flavonoids Accumulation of Liquorice under Nutrient Stress. Frontiers in Plant Science 8: 931.

73. Liu, Jinshan, Zhang, Xiang, Wang, Hui, Xiaoli, Zhaohui, Qiu, Weihong (2018) Long-term nitrogen fertilization impacts soil fungal and bacterial community structures in a dryland soil of Loess Plateau in China. Journal of Soil \& Sediments 18: 1632-1640.

74. Han T, Jia M, Lu B, Qin L (2013) Relationships between endophytic fungi and their hosts: impacts on drug quality of medicinal plants. Planta Medica 79:PN36.

75. Xie W, Hao Z, Yu M, Wu Z, Chen B (2019) Improved phosphorus nutrition by arbuscular mycorrhizal symbiosis as a key factor facilitating glycyrrhizin and liquiritin accumulation in Glycyrrhiza uralensis. Plant and Soil 439: 243-257.

76. Orujei Y, Shabani L, Sharifi-Tehrani M (2013) Induction of glycyrrhizin and total phenolic compound production in licorice by using arbuscular mycorrhizal fungi. Russian Journal of Plant Physiology 60:855-860.

\section{Figures}




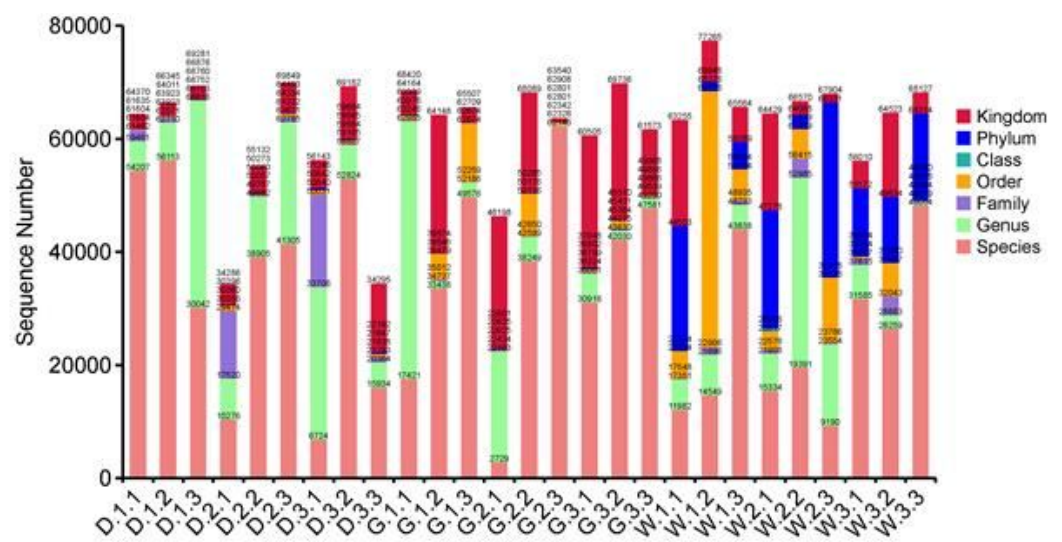

$\mathrm{b}$

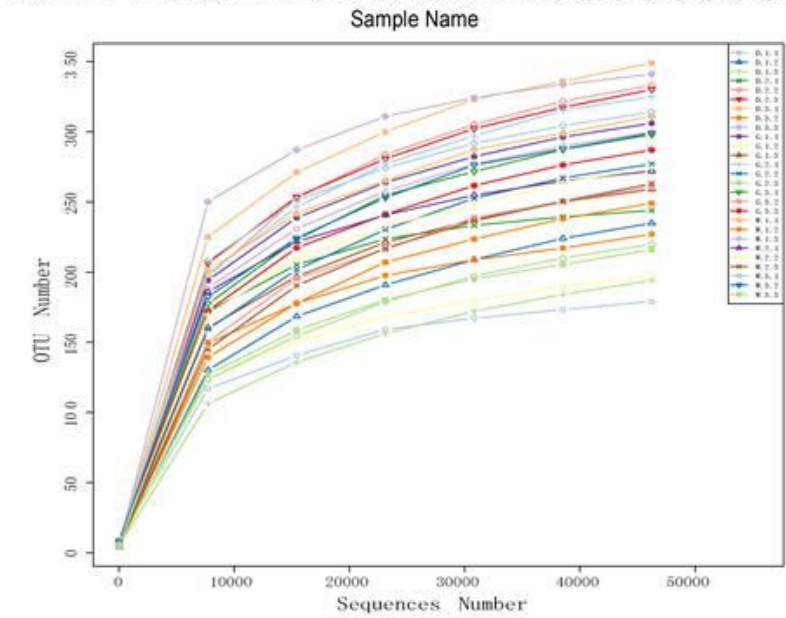

\section{Figure 1}

Distribution of the number of tags on each classification level (Kingdom, Phylum, Class, Order, Family, Genus, Species) (a); Rarefaction curves of fungal community composition (b) Description: Sample Name: D, G and W: Glycyrrhiza inflata, Glycyrrhiza glabra and Glycyrrhiza uralensis; 1, 2 and 3: root depth 0$20 \mathrm{~cm}, 20-40 \mathrm{~cm}$, and $40-60 \mathrm{~cm}$, respectively; the third number representing the replicate number. The rarefaction curves different colors represent different samples. 
a

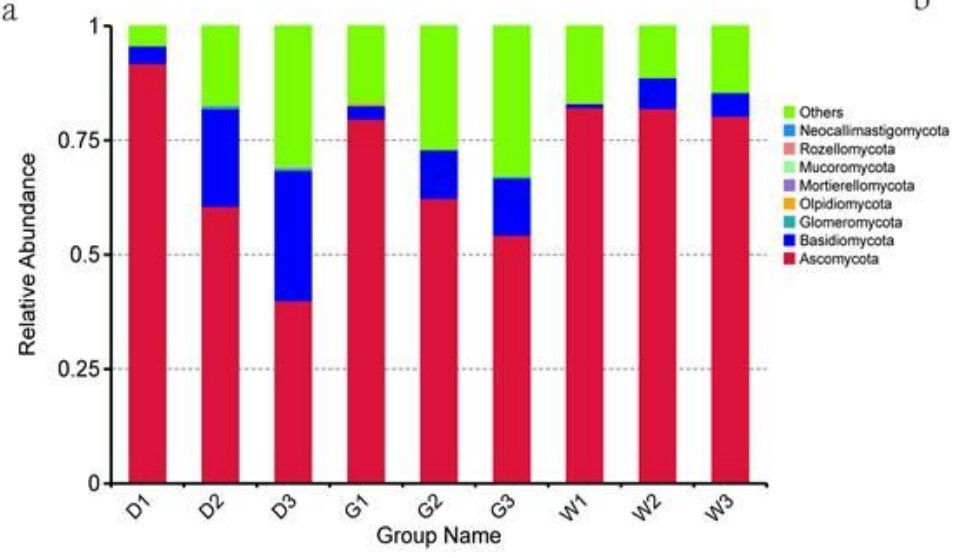

c

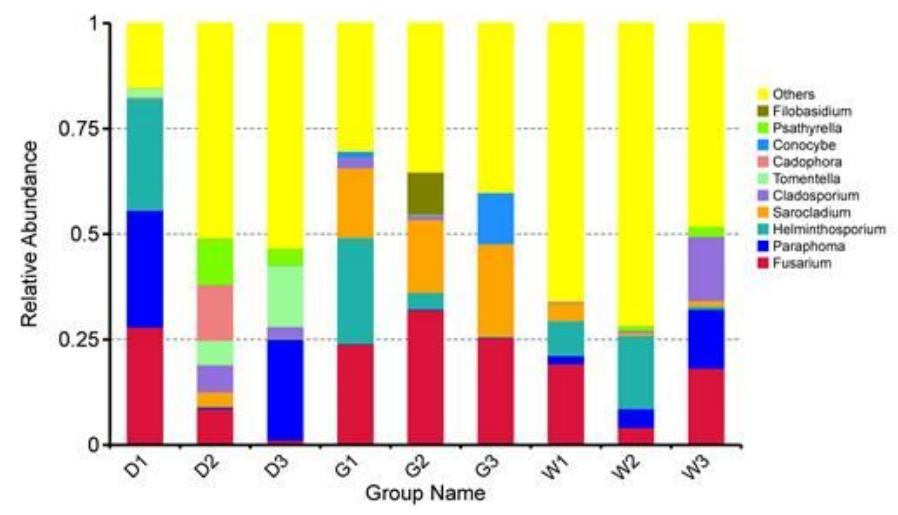

b

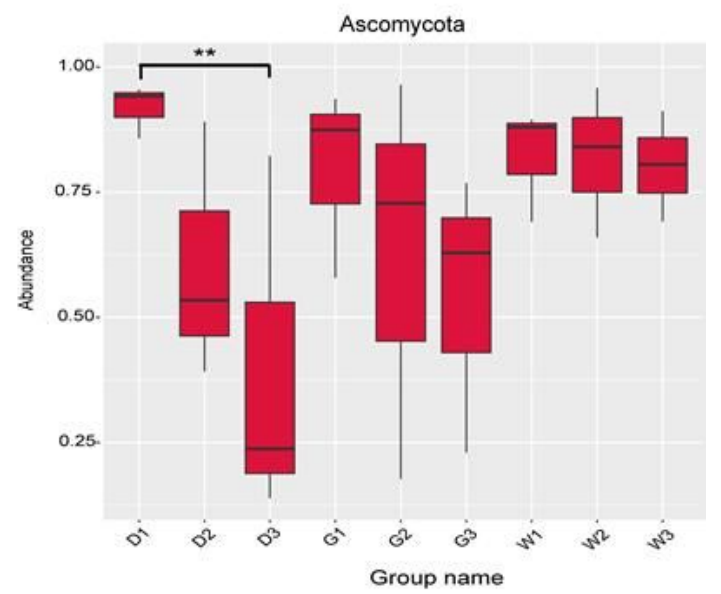

Figure 2

Histograms of relative abundance of the top 10 endophytic fungi at the phyla (a) and genera (c) level of taxonomy; difference analysis at the Phylum classification level (b). Description: Ordinate is the relative abundance; others refers to are sequences with less or not be annotated. Abscissa is the group name: D, G and W: Glycyrrhiza inflata, Glycyrrhiza glabra and Glycyrrhiza uralensis; 1,2 and 3: root depth 0-20cm, 20-40cm, and 40-60cm, respectively. ** means $\mathrm{P}<0.01$.

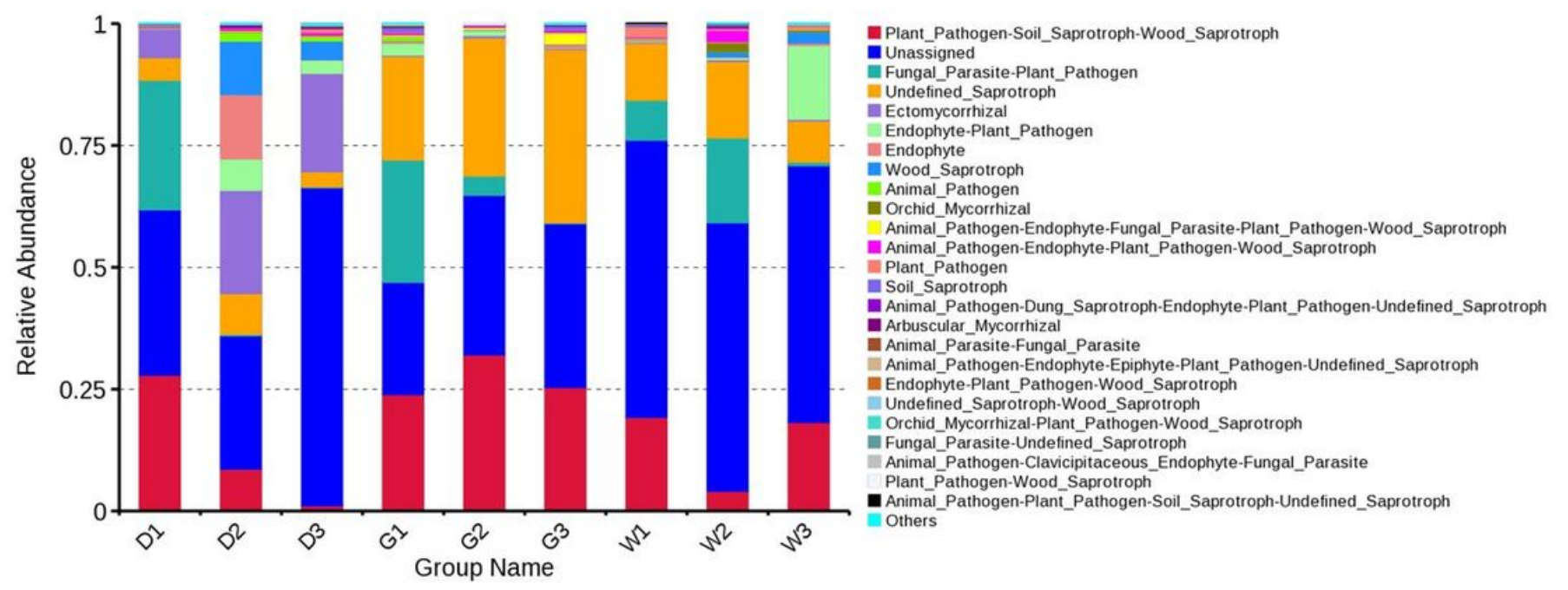

Figure 3

Histograms of relative abundance of the top 25 main ecological function Description: Ordinate is the relative abundance; others refers to are sequences with less or not be annotated. Abscissa is the group name: D, G and W: Glycyrrhiza inflata, Glycyrrhiza glabra and Glycyrrhiza uralensis; 1, 2 and 3: root depth 0$20 \mathrm{~cm}, 20-40 \mathrm{~cm}$, and $40-60 \mathrm{~cm}$, respectively. 
a

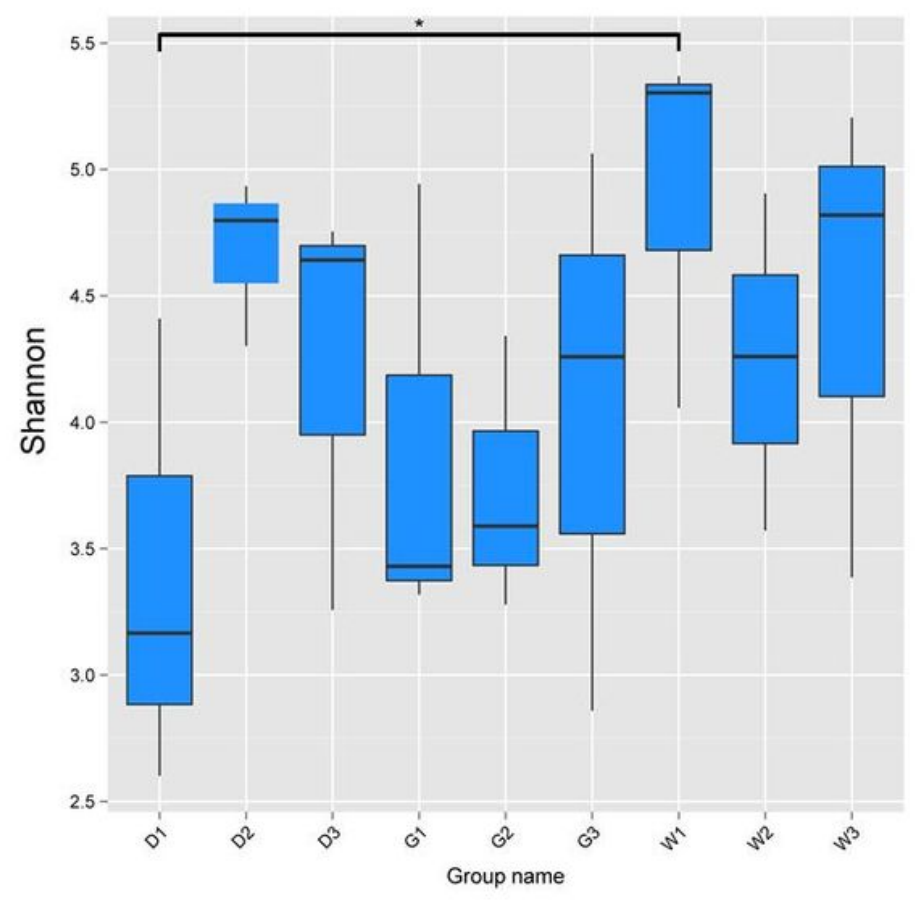

b

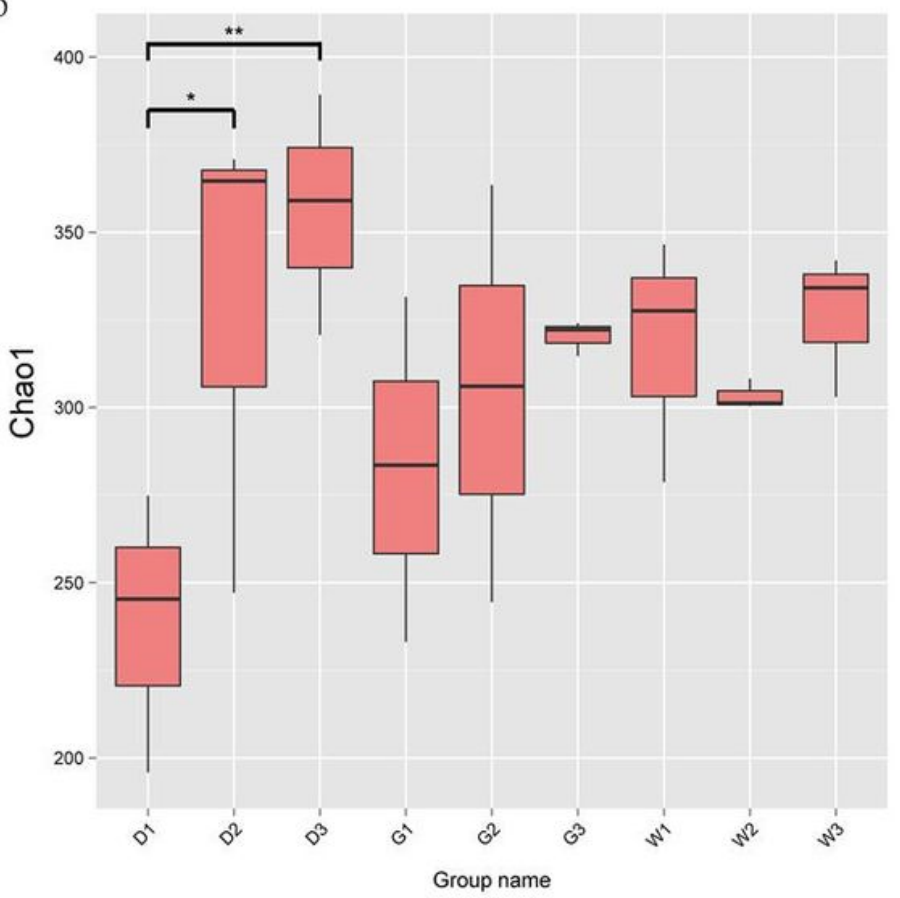

\section{Figure 4}

The significance test of the differences of Alpha Diversity Description: Ordinates are Shannon index (a) and Chao1 index (b), respectively. Abscissa is the group name: D, G and W: Glycyrrhiza inflata, Glycyrrhiza glabra and Glycyrrhiza uralensis; 1,2 and 3: root depth 0-20cm, 20-40cm, and 40-60cm, respectively. The mark * is significance test $p<0.05$.

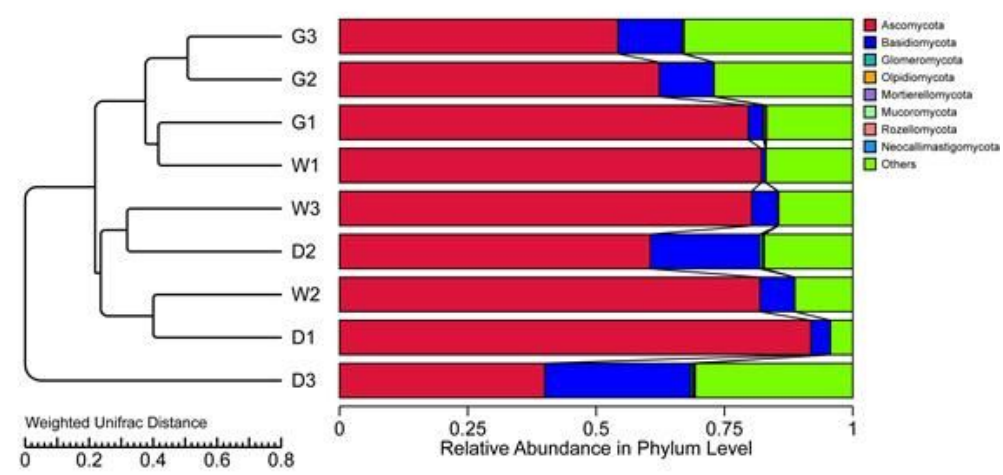

b

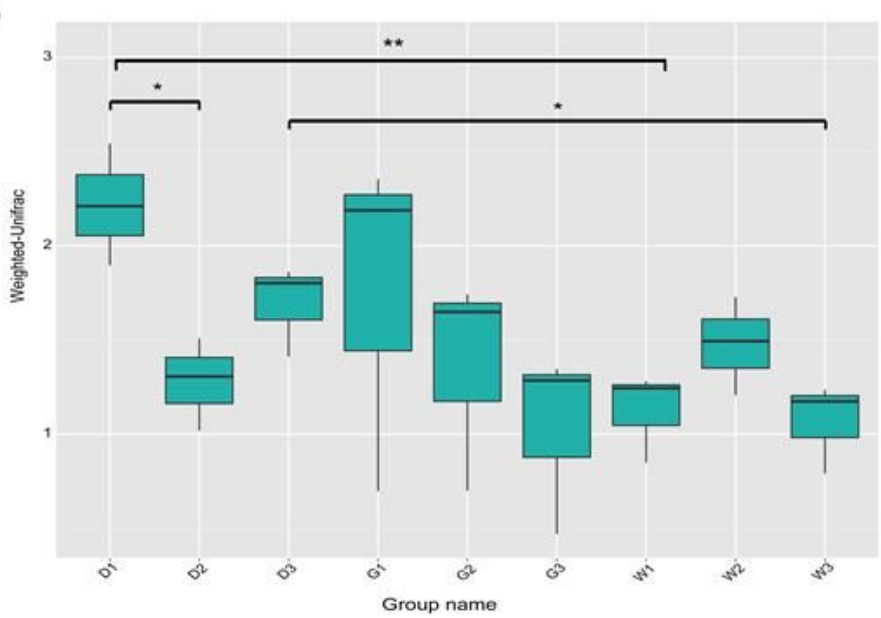

Figure 5 
a

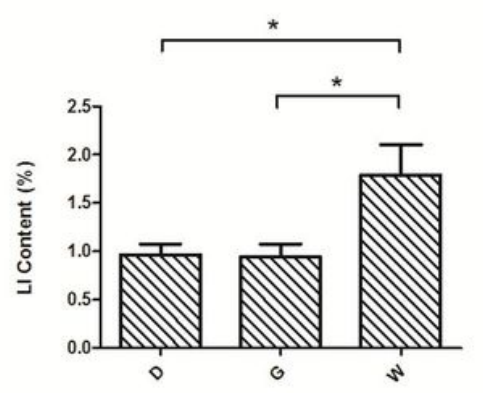

b

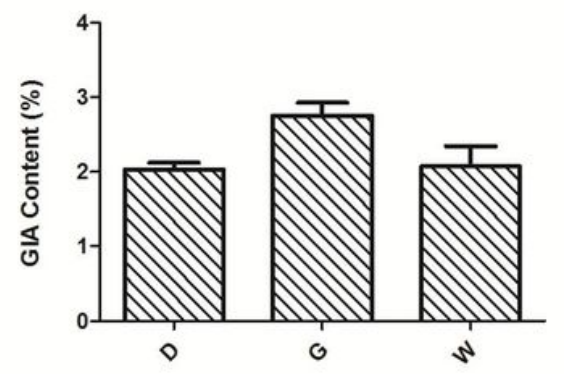

C

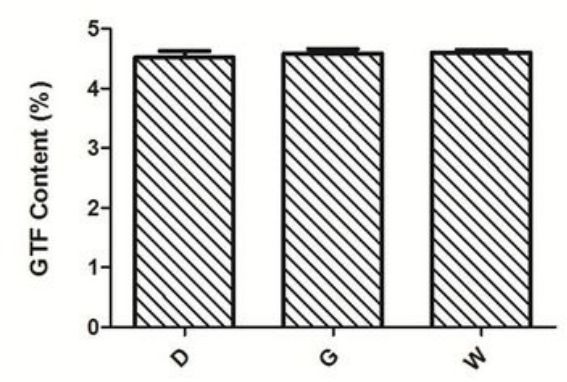

\section{Figure 6}

Effect of main effect plant species on the bioactive compounds of licorice roots Description: Ordinate is the content of LI (a), GIA (b) and GTF (c); abscissa is the group name: D, G and W: Glycyrrhiza inflata, Glycyrrhiza glabra and Glycyrrhiza uralensis, and the mark * is significance test $(p<0.05)$.

\section{Spearman Correlation Heatmap}

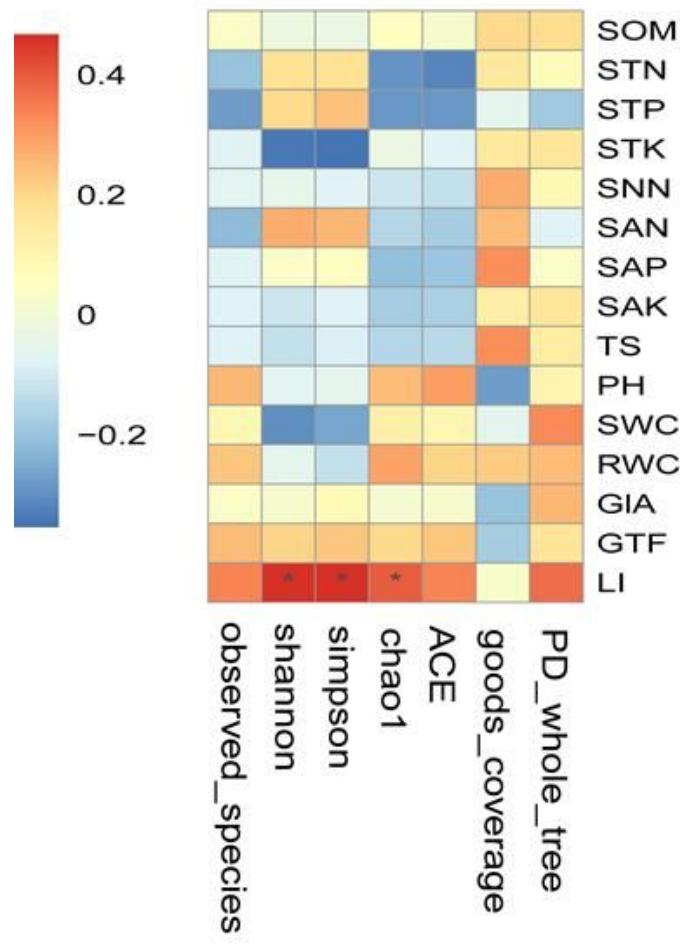

\section{Figure 7}

Heatmaps of Spearman correlation analysis Description: Ordinate is the information of environmental factors, and abscissa is the information of alpha diversity indexes. The correlation coefficient $r$ of Spearman is between -1 and $1, r<0$ is negative correlation, $r>0$ is positive correlation, and the mark * is significance test $(p<0.05)$. 


\section{Spearman Correlation Heatmap}
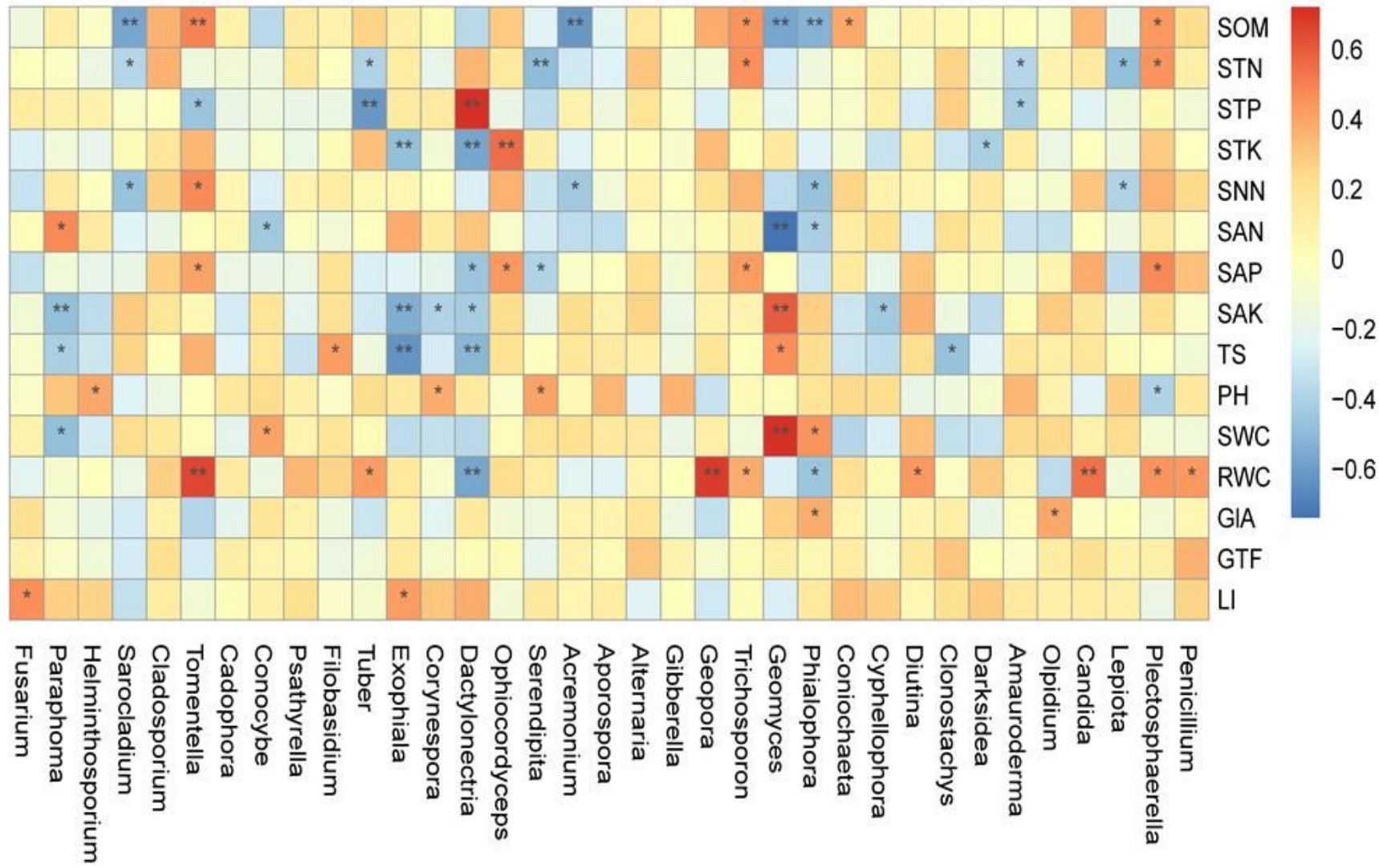

Figure 8

Heatmaps of Spearman correlation analysis Description: Ordinate is the information of environmental factors, and abscissa is the information of species at the genera level of taxonomy. The correlation coefficient $r$ of Spearman is between -1 and $1, r<0$ is negative correlation, $r>0$ is positive correlation, and the mark * is significance test $(p<0.05)$. 


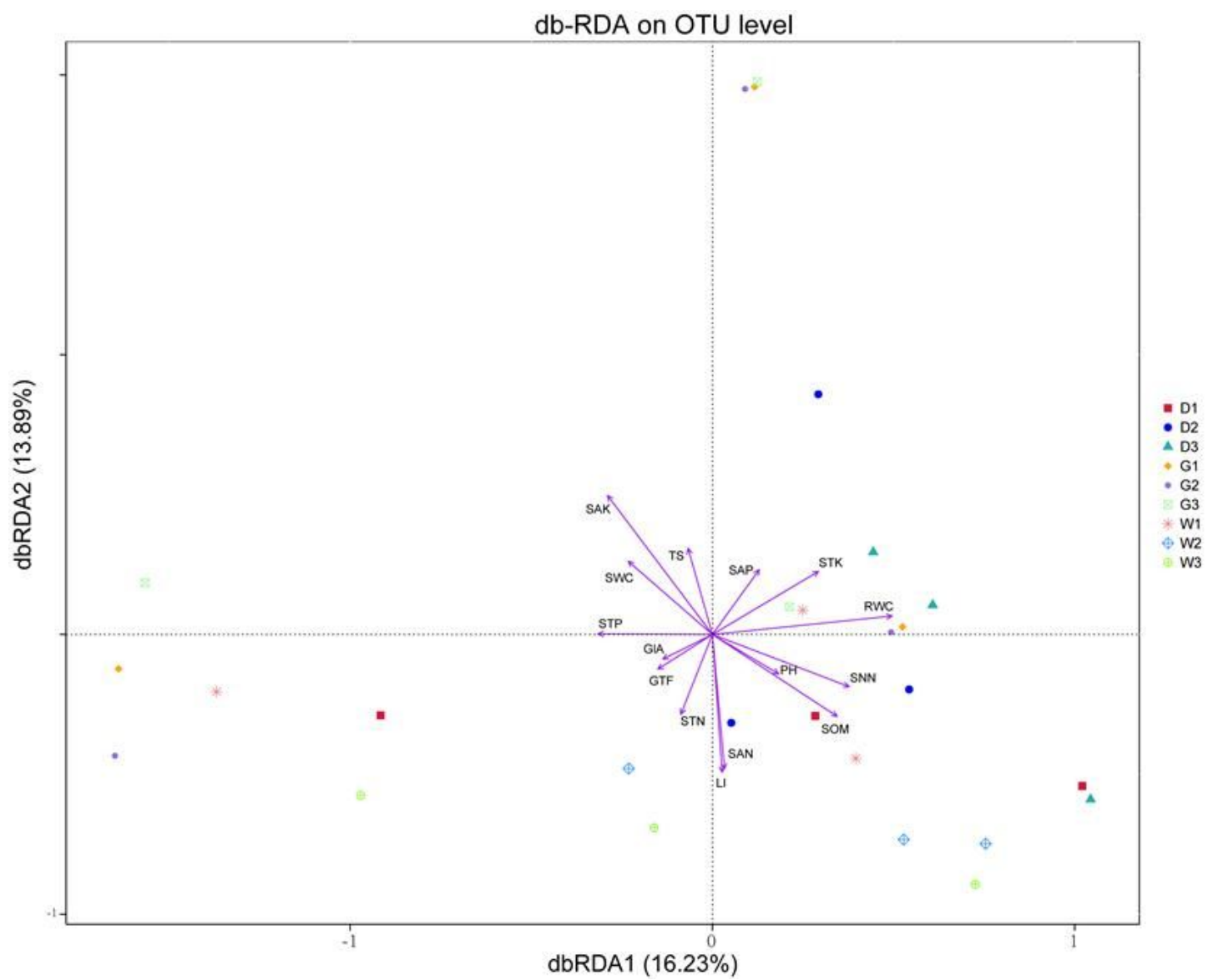

Figure 9

Distance-based redundancy analysis (db-RDA) for all groups Description: Environmental factors are generally represented by arrows. The length of the arrow line represents the degree of correlation between a certain environmental factor and community and species distribution, and the longer the arrow, the greater the correlation. When the angle between the environmental factors is acute, it means that there is a positive correlation between the two environmental factors, while when the angle is obtuse, there is a negative correlation.

\section{Supplementary Files}

This is a list of supplementary files associated with this preprint. Click to download.

- supplementarymaterials.docx

- supplementarymaterials.docx 